\title{
Existence and Monotone Iteration of Positive Pseudosymmetric Solutions for a Third-Order Four-Point BVP with $p$-Laplacian
}

\author{
Dan Li, Libo Wang, and Minghe Pei \\ Department of Mathematics, Beihua University, Jilin 132013, China \\ Correspondence should be addressed to Minghe Pei; peiminghe@163.com
}

Received 25 February 2013; Revised 27 April 2013; Accepted 28 April 2013

Academic Editor: Guoyin Li

Copyright (C) 2013 Dan Li et al. This is an open access article distributed under the Creative Commons Attribution License, which permits unrestricted use, distribution, and reproduction in any medium, provided the original work is properly cited.

We study the existence and monotone iteration of solutions for a third-order four-point boundary value problem with $p$-Laplacian. An existence result of positive, concave, and pseudosymmetric solutions and its monotone iterative scheme are established by using the monotone iterative technique. Meanwhile, as an application of our result, an example is given.

\section{Introduction}

The third-order equations arise in many areas of applied mathematics and physics [1] and thus have been discussed by many authors and many excellent results were obtained; see [1-31] and the references therein. Recently, wide attention has been paid to the third-order boundary value problems with the $p$-Laplace operator. In fact, the third-order equations involving the $p$-Laplace operator can be seen as a generalized model for various physical, natural or physiological phenomena such as the flow of a thin film of viscous fluid over a solid surface, the solitary wave solution of the Korteweg-de Vries equation or a thyroid-pituitary interaction [17].

In 2005, Cabada et al. [7] studied the one-dimensional nonlinear third-order $\phi$-Laplacian equation

$$
-\left(\phi\left(u^{\prime \prime}(t)\right)\right)^{\prime}=f(t, u(t)), \quad t \in[a, b]
$$

with the boundary conditions

$$
u(a)=A, \quad u^{\prime \prime}(a)=B, \quad u^{\prime \prime}(b)=C,
$$

where $\phi: \mathbb{R} \rightarrow \mathbb{R}$ is an increasing homeomorphism with $\phi(0)=0$. By applying the monotone iterative technique based on suitable antimaximum principles, they obtained the existence of extremal solutions for the problem.

In 2006, using the monotone iterative technique, Zhou and $\mathrm{Ma}$ [30] obtained the existence of positive solutions and established a corresponding iterative scheme for the following third-order $p$-Laplacian problem of the form:

$$
\begin{gathered}
\left(\phi_{p}\left(u^{\prime \prime}(t)\right)\right)^{\prime}=q(t) f(t, u(t)), \quad t \in[0,1], \\
u(0)=\sum_{i=1}^{m} \alpha_{i} u\left(\xi_{i}\right), \quad u^{\prime}(\eta)=0, \\
u^{\prime \prime}(1)=\sum_{i=1}^{n} \beta_{i} u^{\prime \prime}\left(\theta_{i}\right) .
\end{gathered}
$$

In 2007, Wang and Ge [26] considered third-order differential equation

$$
\left(\phi\left(u^{\prime \prime}(t)\right)\right)^{\prime}+f\left(t, u(t), u^{\prime}(t), u^{\prime \prime}(t)\right)=0, \quad t \in(0,1)
$$

subject to the following integral boundary conditions:

$$
\begin{gathered}
u(0)=0, \\
u^{\prime}(0)-k_{1} u^{\prime \prime}(0)=\int_{0}^{1} h_{1}(u(s)) \mathrm{d} s, \\
u^{\prime}(1)+k_{2} u^{\prime \prime}(1)=\int_{0}^{1} h_{2}(u(s)) \mathrm{d} s .
\end{gathered}
$$

The existence result to the problem is obtained by applying the method of upper and lower solutions and Leray-Schauder degree theory. 
In 2009, Sun et al. [24] studied the existence of positive solutions for the following third-order $p$-Laplacian problem:

$$
\begin{gathered}
\left(\phi_{p}\left(u^{\prime \prime}(t)\right)\right)^{\prime}=q(t) f\left(t, u(t), u^{\prime}(t), u^{\prime \prime}(t)\right), \quad t \in[0,1], \\
u(0)=\sum_{i=1}^{m} \alpha_{i} u\left(\xi_{i}\right), \quad u^{\prime}(\eta)=0, \quad u^{\prime \prime}(1)=\sum_{i=1}^{n} \beta_{i} u^{\prime \prime}\left(\theta_{i}\right) .
\end{gathered}
$$

By applying a monotone iterative method, the authors obtained the existence of positive solutions for the problem and established iterative schemes for approximating the solutions.

In 2010, Jin and Lu [17] considered the following thirdorder $p$-Laplacian resonant problem of the form:

$$
\begin{gathered}
\left(\phi_{p}\left(x^{\prime \prime}(t)\right)\right)^{\prime}=f\left(t, x(t), x^{\prime}(x), x^{\prime \prime}(t)\right), \quad t \in(0,1), \\
x(0)=0, \quad x^{\prime}(1)=\sum_{i=1}^{m-2} a_{i} x^{\prime}\left(\xi_{i}\right), \quad x^{\prime \prime}(0)=0 .
\end{gathered}
$$

The authors obtained the existence of solutions for the problem by using Mawhin's continuation theorem.

In 2010, by using the fixed point index method, Yang and Yan [31] established the existence of at least one or at least two positive solutions for the following third-order $p$-Laplacian problem:

$$
\begin{gathered}
\left(\phi_{p}\left(u^{\prime \prime}(t)\right)\right)^{\prime}+f(t, u(t))=0, \quad t \in(0,1), \\
\alpha u(0)-\beta u^{\prime}(0)=0, \quad \gamma u(1)+\delta u^{\prime}(1)=0, \\
u^{\prime \prime}(0)=0 .
\end{gathered}
$$

Motivated by the above works and $[32,33]$, in this paper, we consider the existence and monotone iteration of positive, pseudosymmetric solutions of the following third-order fourpoint $p$-Laplacian boundary value problem:

$$
\left(\phi_{p}\left(u^{\prime \prime}(t)\right)\right)^{\prime}+q(t) f\left(t, u(t), u^{\prime}(t)\right)=0, \quad t \in(0,1)
$$

subject to boundary conditions

$$
u(0)=0, \quad u(1)=u(\eta), \quad u^{\prime \prime}\left(\frac{1+\eta}{2}\right)=0,
$$

where $\phi_{p}(s)=|s|^{p-2} s, p>1$, and $\eta \in(0,1)$ be constant. Here $u^{*}(t)$ is said to be a positive solution of BVP (9), (10) if and only if $u^{*}(t)$ is the solution of BVP (9), (10) and satisfies $u^{*}(t)>0$ for $t \in(0,1]$. BVP (9), (10) can model the static deflection of an elastic beam with linear supports at both endpoints.

To the best of our knowledge, the existence results of the pseudosymmetric solutions for the third-order boundary value problem has not been considered.

This work is organized as follows. In Section 2, some notations and preliminaries are introduced. The main results are discussed in Section 3. As applications of our results, an example is given in the last section.

\section{Preliminary}

In this section, we give some definitions and lemmas which help to simplify the presentation of our main result.

Definition 1 (see [32]). Let $u \in C[0,1], \eta \in(0,1)$. One says that $u$ is pseudosymmetric about $\eta$ on $[0,1]$, if $u$ is symmetric on $[\eta, 1]$, that is,

$$
u(t)=u(1+\eta-t), \quad \forall t \in[\eta, 1]
$$

Definition 2. Let $u \in C[0,1], \eta \in(0,1)$. One says that $u$ is pseudo-antisymmetric about $\eta$ on $[0,1]$, if $u$ is antisymmetric on $[\eta, 1]$, that is,

$$
u(t)+u(1+\eta-t)=0, \quad \forall t \in[\eta, 1]
$$

Let the Banach space $E=C^{1}[0,1]$ be endowed with the norm

$$
\|u\|=\max _{0 \leq t \leq 1}\left(u^{2}(t)+u^{\prime 2}(t)\right)^{1 / 2},
$$

and define the cone $P \subset E$ by

$$
\begin{array}{r}
P=\{u \in E: u \text { is nonnegative, concave and } \\
\text { pseudosymmetric about } \eta \text { on }[0,1]\},
\end{array}
$$

and let

$$
\bar{P}_{a}=\{u \in P:\|u\| \leq a\} .
$$

For convenience, we consider the following.

$\left(\mathrm{H}_{0}\right) q(t)$ is a nonnegative continuous function defined on $(0,1), q(t) \neq \equiv 0$ on any subinterval of $(0,1)$. In addition, $\int_{0}^{1} q(t) \mathrm{d} t<+\infty$ and $q(t)$ is pseudosymmetric about $\eta$ on $[0,1]$.

$\left(\mathrm{H}_{1}\right) f(t, u, v):[0,1] \times[0,+\infty) \times \mathbb{R} \rightarrow \mathbb{R}$ is continuous and

$$
f(t, u, v) \geq 0, \quad \forall(t, u, v) \in\left[0, \frac{1+\eta}{2}\right] \times[0,+\infty) \times \mathbb{R}
$$

$\left(\mathrm{H}_{2}\right) f(t, u, v)+f(1+\eta-t, u,-v)=0$ for all $(t, u, v) \epsilon$ $[\eta, 1] \times[0,+\infty) \times \mathbb{R}$

$\left(\mathrm{H}_{3}\right) f(t, 0,0) \not \equiv 0$ on $[0,1]$. 
Now, we define an operator $T: C^{1}[0,1] \rightarrow C^{1}[0,1]$ as follows: for $u \in C^{1}[0,1]$,

$$
(T u)(t)=\left\{\begin{array}{r}
\int_{0}^{t} \int_{\tau}^{(1+\eta) / 2} \phi_{p}^{-1}\left(\int_{s}^{(1+\eta) / 2} q(r)\right. \\
\left.\quad \times f\left(r, u(r), u^{\prime}(r)\right) \mathrm{d} r\right) \mathrm{d} s \mathrm{~d} \tau, \\
\int_{0}^{\eta} \int_{\tau}^{(1+\eta) / 2} \phi_{p}^{-1}\left(\int_{s}^{(1+\eta) / 2} q(r)\right. \\
\left.\times f\left(r, u(r), u^{\prime}(r)\right) \mathrm{d} r\right) \mathrm{d} s \mathrm{~d} \tau \\
-\int_{t}^{1} \int_{(1+\eta) / 2}^{\tau} \phi_{p}^{-1}\left(\int_{(1+\eta) / 2}^{s} q(r)\right. \\
\left.\times f\left(r, u(r), u^{\prime}(r)\right) \mathrm{d} r\right) \mathrm{d} s \mathrm{~d} \tau, \\
\frac{1+\eta}{2} \leq t \leq 1 .
\end{array}\right.
$$

Obviously under assumptions $\left(\mathrm{H}_{0}\right)$ and $\left(\mathrm{H}_{1}\right)$, the operator $T$ is well defined and it is easy to verify that BVP (9), (10) has a solution if and only if $T: C^{1}[0,1] \rightarrow C^{1}[0,1]$ has a fixed point.

The next lemmas are some properties of the operator $T$.

Lemma 3. Assume that $\left(H_{0}\right),\left(H_{1}\right)$, and $\left(H_{2}\right)$ hold. Then TP $\subset$ $P$.

Proof. From the definition of $T$, it is easy to check that $T u$ is nonnegative on $[0,1]$ and satisfies (10) for all $u \in P$. Furthermore, since

$$
(T u)^{\prime \prime}(t)=\left\{\begin{array}{c}
-\phi_{p}^{-1}\left(\int_{t}^{(1+\eta) / 2} q(r) f\left(r, u(r), u^{\prime}(r)\right) \mathrm{d} r\right) \\
\leq 0, \quad 0 \leq t \leq \frac{1+\eta}{2}, \\
\phi_{p}^{-1}\left(\int_{(1+\eta) / 2}^{t} q(r) f\left(r, u(r), u^{\prime}(r)\right) \mathrm{d} r\right) \\
\leq 0, \quad \frac{1+\eta}{2} \leq t \leq 1,
\end{array}\right.
$$

it follows that $T u$ is concave on $[0,1]$.

Next we prove that $T u$ is pseudosymmetric about $\eta$ on $[0,1]$. In fact, if $t \in[\eta,(1+\eta) / 2]$, then $1+\eta-t \in[(1+\eta) / 2,1]$, and it follows that

$$
\begin{aligned}
& (\mathrm{Tu})(1+\eta-t) \\
& \begin{array}{r}
\int_{0}^{\eta} \int_{\tau}^{(1+\eta) / 2} \phi_{p}^{-1} \\
\quad \times\left(\int_{s}^{(1+\eta) / 2} q(r) f\left(r, u(r), u^{\prime}(r)\right) \mathrm{d} r\right) \mathrm{d} s \mathrm{~d} \tau \\
-\int_{1+\eta-t}^{1} \int_{(1+\eta) / 2}^{\tau} \phi_{p}^{-1} \\
\quad \times\left(\int_{(1+\eta) / 2}^{s} q(r) f\left(r, u(r), u^{\prime}(r)\right) \mathrm{d} r\right) \mathrm{d} s \mathrm{~d} \tau
\end{array}
\end{aligned}
$$

$$
\begin{array}{r}
=\int_{0}^{\eta} \int_{\tau}^{(1+\eta) / 2} \phi_{p}^{-1} \\
\quad \times\left(\int_{s}^{(1+\eta) / 2} q(r) f\left(r, u(r), u^{\prime}(r)\right) \mathrm{d} r\right) \mathrm{d} s \mathrm{~d} \tau \\
+\int_{1}^{\eta} \int_{(1+\eta) / 2}^{\tau} \phi_{p}^{-1} \\
\quad \times\left(\int_{(1+\eta) / 2}^{s} q(r) f\left(r, u(r), u^{\prime}(r)\right) \mathrm{d} r\right) \mathrm{d} s \mathrm{~d} \tau \\
+\int_{\eta}^{t} \int_{(1+\eta) / 2}^{\tau} \phi_{p}^{-1} \\
\times\left(\int_{(1+\eta) / 2}^{s} q(r) f\left(r, u(r), u^{\prime}(r)\right) \mathrm{d} r\right) \mathrm{d} s \mathrm{~d} \tau \\
+\int_{t}^{1+\eta-t} \int_{(1+\eta) / 2}^{\tau} \phi_{p}^{-1} \\
\times\left(\int_{(1+\eta) / 2}^{s} q(r) f\left(r, u(r), u^{\prime}(r)\right) \mathrm{d} r\right) \mathrm{d} s \mathrm{~d} \tau .
\end{array}
$$

Also since $u$ is pseudosymmetric about $\eta$ on $[0,1]$, that is, $u(t)=u(1+\eta-t)$ for $t \in[\eta, 1]$, then

$$
u^{\prime}(t)=-u^{\prime}(1+\eta-t), \quad t \in[\eta, 1] .
$$

Thus, for all $t \in[\eta, 1]$, from $\left(\mathrm{H}_{2}\right)$, we have

$$
\begin{aligned}
q(r) f & \left(r, u(r), u^{\prime}(r)\right) \\
= & -q(1+\eta-r) f\left(1+\eta-r, u(r),-u^{\prime}(r)\right) \\
= & -q(1+\eta-r) \\
& \times f\left(1+\eta-r, u(1+\eta-r), u^{\prime}(1+\eta-r)\right) .
\end{aligned}
$$

Hence $q(r) f\left(r, u(r), u^{\prime}(r)\right)$ is pseudo-antisymmetric about $\eta$ on $[0,1]$, and thus $\int_{(1+\eta) / 2}^{s} q(r) f\left(r, u(r), u^{\prime}(r)\right) \mathrm{d} r$ is pseudosymmetric about $\eta$ on $[0,1]$. Furthermore $\phi_{p}^{-1}\left(\int_{(1+\eta) / 2}^{s} q(r) f\left(r, u(r), u^{\prime}(r)\right) \mathrm{d} r\right)$ is pseudosymmetric about $\eta$ on $[0,1]$. Thus the function $\int_{(1+\eta) / 2}^{\tau} \phi_{p}^{-1}$ $\left(\int_{(1+\eta) / 2}^{s} q(r) f\left(r, u(r), u^{\prime}(r)\right) \mathrm{d} r\right) \mathrm{d} s$ is pseudo-antisymmetric about $\eta$ on $[0,1]$, and hence

$$
\begin{aligned}
& \int_{1}^{\eta} \int_{(1+\eta) / 2}^{\tau} \phi_{p}^{-1}\left(\int_{(1+\eta) / 2}^{s} q(r) f\left(r, u(r), u^{\prime}(r)\right) \mathrm{d} r\right) \mathrm{d} s \mathrm{~d} \tau \\
& \quad=0 .
\end{aligned}
$$


Using the similar technique, we can get

$$
\begin{aligned}
\int_{t}^{1+\eta-t} & \int_{(1+\eta) / 2}^{\tau} \phi_{p}^{-1}\left(\int_{(1+\eta) / 2}^{s} q(r) f\left(r, u(r), u^{\prime}(r)\right) \mathrm{d} r\right) \mathrm{d} s \mathrm{~d} \tau \\
\quad= & 0 .
\end{aligned}
$$

From (19), (22), and (23), it follows that

$$
\begin{aligned}
& (\text { Tu) }(1+\eta-t) \\
& =\int_{0}^{\eta} \int_{\tau}^{(1+\eta) / 2} \phi_{p}^{-1} \\
& \quad \times\left(\int_{s}^{(1+\eta) / 2} q(r) f\left(r, u(r), u^{\prime}(r)\right) \mathrm{d} r\right) \mathrm{d} s \mathrm{~d} \tau \\
& +\int_{\eta}^{t} \int_{(1+\eta) / 2}^{\tau} \phi_{p}^{-1} \\
& \times\left(\int_{(1+\eta) / 2}^{s} q(r) f\left(r, u(r), u^{\prime}(r)\right) \mathrm{d} r\right) \mathrm{d} s \mathrm{~d} \tau \\
& =\int_{0}^{t} \int_{(1+\eta) / 2}^{\tau} \phi_{p}^{-1} \\
& \times\left(\int_{(1+\eta) / 2}^{s} q(r) f\left(r, u(r), u^{\prime}(r)\right) \mathrm{d} r\right) \mathrm{d} s \mathrm{~d} \tau \\
& =(T u)(t), \quad t \in\left[\eta, \frac{1+\eta}{2}\right] .
\end{aligned}
$$

If $t \in[(1+\eta) / 2,1]$, then $1+\eta-t \in[\eta,(1+\eta) / 2]$. From (24), it follows that

$$
\begin{aligned}
(T u)(1+\eta-t) & =(T u)(1+\eta-(1+\eta-t)) \\
& =(T u)(t), \quad t \in\left[\frac{1+\eta}{2}, 1\right] .
\end{aligned}
$$

This together with (24) implies that

$$
(T u)(t)=(T u)(1+\eta-t), \quad t \in[\eta, 1] .
$$

In summary, $T u \in P$, and then $T P \subset P$.

The following lemma can be easily verified by a standard argument.

Lemma 4. Assume that $\left(H_{0}\right),\left(H_{1}\right)$, and $\left(H_{2}\right)$ hold. Then $T$ : $P \rightarrow P$ is completely continuous.

Lemma 5. Assume that $\left(H_{0}\right),\left(H_{1}\right)$, and $\left(H_{2}\right)$ hold. Suppose also that there exists $a>0$ such that for $0 \leq t \leq(1+\eta) / 2$, $0 \leq u_{1} \leq u_{2} \leq a, 0 \leq\left|v_{1}\right| \leq\left|v_{2}\right| \leq a$,

$$
f\left(t, u_{1}, v_{1}\right) \leq f\left(t, u_{2}, v_{2}\right) \text {. }
$$

Then for $u_{1}, u_{2} \in \bar{P}_{a}$ with

$$
u_{1}(t) \leq u_{2}(t), \quad\left|u_{1}^{\prime}(t)\right| \leq\left|u_{2}^{\prime}(t)\right|, \quad t \in[0,1],
$$

we have

$$
\begin{array}{r}
\left(T u_{1}\right)(t) \leq\left(T u_{2}\right)(t), \quad\left|\left(T u_{1}\right)^{\prime}(t)\right| \leq\left|\left(T u_{2}\right)^{\prime}(t)\right|, \\
t \in[0,1] .
\end{array}
$$

Proof. First we prove that, for all $t \in[0,(1+\eta) / 2]$,

$$
\left(T u_{1}\right)(t) \leq\left(T u_{2}\right)(t), \quad\left|\left(T u_{1}\right)^{\prime}(t)\right| \leq\left|\left(T u_{2}\right)^{\prime}(t)\right| .
$$

From assumptions, we have

$$
f\left(r, u_{1}(r), u_{1}^{\prime}(r)\right) \leq f\left(r, u_{2}(r), u_{2}^{\prime}(r)\right), \quad r \in\left[0, \frac{1+\eta}{2}\right],
$$

and hence

$$
\begin{aligned}
& \int_{s}^{(1+\eta) / 2} f\left(r, u_{1}(r), u_{1}^{\prime}(r)\right) \mathrm{d} r \\
& \quad \leq \int_{s}^{(1+\eta) / 2} f\left(r, u_{2}(r), u_{2}^{\prime}(r)\right) \mathrm{d} r, \quad s \in\left[0, \frac{1+\eta}{2}\right] .
\end{aligned}
$$

Since $\phi_{p}^{-1}$ is strictly increasing on $\mathbb{R}$, then for all $s \in[0,(1+$ $\eta) / 2$ ], we have

$$
\begin{aligned}
& \phi_{p}^{-1}\left(\int_{s}^{(1+\eta) / 2} f\left(r, u_{1}(r), u_{1}^{\prime}(r)\right) \mathrm{d} r\right) \\
& \quad \leq \phi_{p}^{-1}\left(\int_{s}^{(1+\eta) / 2} f\left(r, u_{2}(r), u_{2}^{\prime}(r)\right) \mathrm{d} r\right) .
\end{aligned}
$$

Thus for $t \in[0,(1+\eta) / 2]$,

$$
\begin{aligned}
&\left(T u_{1}\right)(t)-\left(T u_{2}\right)(t) \\
&=\int_{0}^{t} \int_{\tau}^{(1+\eta) / 2}\left[\phi_{p}^{-1}\left(\int_{s}^{(1+\eta) / 2} q(r) f\left(r, u_{1}(r), u_{1}^{\prime}(r)\right) \mathrm{d} r\right)\right. \\
&\left.\quad-\phi_{p}^{-1}\left(\int_{s}^{(1+\eta) / 2} q(r) f\left(r, u_{2}(r), u_{2}^{\prime}(r)\right) \mathrm{d} r\right)\right] \mathrm{d} s \mathrm{~d} \tau
\end{aligned}
$$

$\leq 0$

$$
\begin{aligned}
\left|\left(T u_{1}\right)^{\prime}(t)\right|-\left|\left(T u_{2}\right)^{\prime}(t)\right| & \\
=\int_{t}^{(1+\eta) / 2} & {\left[\phi_{p}^{-1}\left(\int_{s}^{(1+\eta) / 2} q(r) f\left(r, u_{1}(r), u_{1}^{\prime}(r)\right) \mathrm{d} r\right)\right.} \\
& \left.-\phi_{p}^{-1}\left(\int_{s}^{(1+\eta) / 2} q(r) f\left(r, u_{2}(r), u_{2}^{\prime}(r)\right) \mathrm{d} r\right)\right] \mathrm{d} s
\end{aligned}
$$

$\leq 0$.

Therefore, (30) holds for $t \in[0,(1+\eta) / 2]$.

Next we prove that $(30)$ holds for $t \in[(1+\eta) / 2,1]$. In fact, if $t \in[(1+\eta) / 2,1]$, then $1+\eta-t \in[0,(1+\eta) / 2]$, and hence 
from the fact that $T u_{1}$ and $T u_{2}$ are pseudosymmetric about $\eta$ on $[0,1]$, it follows that, for $t \in[(1+\eta) / 2,1]$,

$$
\begin{aligned}
\left(T u_{1}\right)(t)-\left(T u_{2}\right)(t)= & \left(T u_{1}\right)(1+\eta-t) \\
& -\left(T u_{2}\right)(1+\eta-t) \\
\leq & 0, \\
\left|\left(T u_{1}\right)^{\prime}(t)\right|-\left|\left(T u_{2}\right)^{\prime}(t)\right|= & \left|\left(T u_{1}\right)^{\prime}(1+\eta-t)\right| \\
& -\left|\left(T u_{2}\right)^{\prime}(1+\eta-t)\right|
\end{aligned}
$$$$
\leq 0 \text {. }
$$

In summary,

$$
\begin{array}{r}
\left(T u_{1}\right)(t) \leq\left(T u_{2}\right)(t), \quad\left|\left(T u_{1}\right)^{\prime}(t)\right| \leq\left|\left(T u_{2}\right)^{\prime}(t)\right| \\
t \in[0,1] .
\end{array}
$$

Now, we introduce some notations as follows:

$$
\begin{gathered}
A_{1}=\int_{0}^{(1+\eta) / 2} \phi_{p}^{-1}\left(\int_{s}^{(1+\eta) / 2} q(r) \mathrm{d} r\right) \mathrm{d} s, \\
A_{2}=\phi_{p}^{-1}\left(\int_{0}^{(1+\eta) / 2} q(r) \mathrm{d} r\right), \\
A=\max \left\{\sqrt{2} A_{1}, \sqrt{2} A_{2}\right\}=\sqrt{2} A_{2} .
\end{gathered}
$$

Lemma 6. Assume that $\left(H_{0}\right),\left(H_{1}\right)$, and $\left(H_{2}\right)$ hold. Suppose also that there exists $a>0$ such that for $0 \leq t \leq(1+\eta) / 2$, $0 \leq u_{1} \leq u_{2} \leq a, 0 \leq\left|v_{1}\right| \leq\left|v_{2}\right| \leq a$,

$$
\begin{gathered}
f\left(t, u_{1}, v_{1}\right) \leq f\left(t, u_{2}, v_{2}\right), \\
\max _{0 \leq t \leq(1+\eta) / 2} f(t, a, a) \leq \phi_{p}\left(\frac{a}{A}\right) .
\end{gathered}
$$

Then $T: \bar{P}_{a} \rightarrow \bar{P}_{a}$.

Proof. Define two functionals on $E$ as follows:

$$
\alpha(u):=\max _{0 \leq t \leq 1}|u(t)|, \quad \beta(u):=\max _{0 \leq t \leq 1}\left|u^{\prime}(t)\right| .
$$

Then

$$
\|u\| \leq \sqrt{2} \max \{\alpha(u), \beta(u)\}
$$

If $u \in \bar{P}_{a}$, then

$$
\begin{gathered}
0 \leq u(t) \leq \max _{0 \leq t \leq 1}|u(t)| \leq\|u\| \leq a, \quad t \in[0,1], \\
0 \leq\left|u^{\prime}(t)\right| \leq \max _{0 \leq t \leq 1}\left|u^{\prime}(t)\right| \leq\|u\| \leq a, \quad t \in[0,1] .
\end{gathered}
$$

From the assumptions, for all $t \in[0,(1+\eta) / 2]$,

$$
\begin{aligned}
0 & \leq f\left(t, u(t), u^{\prime}(t)\right) \leq f(t, a, a) \\
& \leq \max _{0 \leq t \leq(1+\eta) / 2} f(t, a, a) \leq \phi_{p}\left(\frac{a}{A}\right) .
\end{aligned}
$$

Then,

$$
\begin{aligned}
& \alpha(T u) \\
& =\max _{0 \leq t \leq 1}|(T u)(t)|=(T u)\left(\frac{1+\eta}{2}\right) \\
& =\int_{0}^{(1+\eta) / 2} \int_{\tau}^{(1+\eta) / 2} \phi_{p}^{-1} \\
& \times\left(\int_{s}^{(1+\eta) / 2} q(r) f\left(r, u(r), u^{\prime}(r)\right) \mathrm{d} r\right) \mathrm{d} s \mathrm{~d} \tau \\
& \leq \int_{0}^{(1+\eta) / 2} \int_{\tau}^{(1+\eta) / 2} \phi_{p}^{-1} \\
& \times\left(\int_{s}^{(1+\eta) / 2} q(r) \phi_{p}\left(\frac{a}{A}\right) \mathrm{d} r\right) \mathrm{d} s \mathrm{~d} \tau \\
& \leq A_{1} \cdot \frac{a}{A} \leq \frac{\sqrt{2}}{2} a, \\
& \beta(T u) \\
& =\max _{0 \leq t \leq 1}\left|(T u)^{\prime}(t)\right|=(T u)^{\prime}(0) \\
& =\int_{0}^{(1+\eta) / 2} \phi_{p}^{-1}\left(\int_{s}^{(1+\eta) / 2} q(r) f\left(r, u(r), u^{\prime}(r)\right) \mathrm{d} r\right) \mathrm{d} s \\
& \leq \int_{0}^{(1+\eta) / 2} \phi_{p}^{-1}\left(\int_{s}^{(1+\eta) / 2} q(r) \phi_{p}\left(\frac{a}{A}\right) \mathrm{d} r\right) \mathrm{d} s \\
& \leq A_{2} \cdot \frac{a}{A} \leq \frac{\sqrt{2}}{2} a \text {. }
\end{aligned}
$$

So we have

$$
\|T u\| \leq \sqrt{2} \max \{\alpha(T u), \beta(T u)\} \leq \sqrt{2} \cdot \frac{\sqrt{2}}{2} a=a .
$$

Thus $T: \bar{P}_{a} \rightarrow \bar{P}_{a}$.

\section{Main Result}

Now we establish existence result of positive, concave, and pseudosymmetric solutions and its monotone iterative scheme for BVP (9), (10).

Theorem 7. Assume that $\left(H_{0}\right),\left(H_{1}\right),\left(H_{2}\right)$, and $\left(H_{3}\right)$ hold. Suppose also that there exists $a>0$ such that for $0 \leq t \leq$ $(1+\eta) / 2,0 \leq u_{1} \leq u_{2} \leq a, 0 \leq\left|v_{1}\right| \leq\left|v_{2}\right| \leq a$,

$$
\begin{gathered}
f\left(t, u_{1}, v_{1}\right) \leq f\left(t, u_{2}, v_{2}\right), \\
\max _{0 \leq t \leq(1+\eta) / 2} f(t, a, a) \leq \phi_{p}\left(\frac{a}{A}\right) .
\end{gathered}
$$


Then BVP (9), (10) has two positive, concave, and pseudosymmetric solutions $w^{*}$ and $v^{*}$ with

$$
\begin{gathered}
0<w^{*}(t) \leq \frac{\sqrt{2}}{2} a, \quad t \in(0,1], \\
\lim _{n \rightarrow \infty} w_{n}=\lim _{n \rightarrow \infty} T^{n} w_{0}=w^{*}\left(\text { in } C^{1} \text { norm }\right), \\
0<v^{*}(t) \leq a, \quad t \in(0,1], \\
\lim _{n \rightarrow \infty} v_{n}=\lim _{n \rightarrow \infty} T^{n} v_{0}=v^{*}\left(\text { in } C^{1} \text { norm }\right),
\end{gathered}
$$

where

$$
\begin{gathered}
w_{0}(t)=\left\{\begin{array}{cc}
\frac{\sqrt{2}}{2} a t, & 0 \leq t \leq \frac{1+\eta}{2}, \\
\frac{\sqrt{2}}{2} a(1+\eta-t), & \frac{1+\eta}{2} \leq t \leq 1 ;
\end{array}\right. \\
(\mathrm{Tu})(t)=\left\{\begin{array}{c}
\int_{0}^{t} \int_{\tau}^{(1+\eta) / 2} \phi_{p}^{-1}\left(\int_{s}^{(1+\eta) / 2} q(r)\right. \\
\left.\quad \times f\left(r, u(r), u^{\prime}(r)\right) \mathrm{dr}\right) \mathrm{ds} \mathrm{d} \tau, \\
\int_{0}^{\eta} \int_{\tau}^{(1+\eta) / 2} \phi_{p}^{-1}\left(\int_{s}^{(1+\eta) / 2} q(r)\right. \\
\left.\quad \times f\left(r, u(r), u^{\prime}(r)\right) \mathrm{dr}\right) \mathrm{ds} \mathrm{d} \tau \\
-\int_{t}^{1} \int_{(1+\eta) / 2}^{\tau} \phi_{p}^{-1}\left(\int_{(1+\eta) / 2}^{s} q(r)\right. \\
\left.\times f\left(r, u(r), u^{\prime}(r)\right) \mathrm{dr}\right) \mathrm{ds} \mathrm{d} \tau,
\end{array}\right.
\end{gathered}
$$

Proof. Let

$$
\begin{gathered}
w_{0}(t)=\left\{\begin{array}{cc}
\frac{\sqrt{2}}{2} a t, & 0 \leq t \leq \frac{1+\eta}{2}, \\
\frac{\sqrt{2}}{2} a(1+\eta-t), & \frac{1+\eta}{2} \leq t \leq 1,
\end{array}\right. \\
w_{1}(t)=\left\{\begin{array}{cc}
\int_{0}^{t} \int_{\tau}^{(1+\eta) / 2} \phi_{p}^{-1}\left(\int_{s}^{(1+\eta) / 2} q(r)\right. \\
\left.\quad \times f\left(r, w_{0}(r), w_{0}^{\prime}(r)\right) \mathrm{d} r\right) \mathrm{d} s \mathrm{~d} \tau, \\
\int_{0}^{\eta} \int_{\tau}^{(1+\eta) / 2} \phi_{p}^{-1}\left(\int_{s}^{(1+\eta) / 2} q(r)\right. \\
\left.\quad \times f\left(r, w_{0}(r), w_{0}^{\prime}(r)\right) \mathrm{d} r\right) \mathrm{d} s \mathrm{~d} \tau, \\
-\int_{t}^{1} \int_{(1+\eta) / 2}^{\tau} \phi_{p}^{-1}\left(\int_{(1+\eta) / 2}^{s} q(r)\right. \\
\left.\quad \times f\left(r, w_{0}(r), w_{0}^{\prime}(r)\right) \mathrm{d} r\right) \mathrm{d} s \mathrm{~d} \tau,
\end{array}\right. \\
\\
\quad \frac{1+\eta}{2} \leq t \leq 1 .
\end{gathered}
$$

Then $w_{1}(t) \in C^{1}[0,(1+\eta) / 2] \cap C^{1}[(1+\eta) / 2,1]$.

Next we prove that

$$
\begin{aligned}
& \lim _{t \rightarrow((1+\eta) / 2)^{-}} w_{1}(t)=\lim _{t \rightarrow((1+\eta) / 2)^{+}} w_{1}(t), \\
& \lim _{t \rightarrow((1+\eta) / 2)^{-}} w_{1}^{\prime}(t)=\lim _{t \rightarrow((1+\eta) / 2)^{+}} w_{1}^{\prime}(t) .
\end{aligned}
$$

In fact, from $\left(\mathrm{H}_{2}\right)$ it follows that

$$
\begin{aligned}
& \lim _{t \rightarrow((1+\eta) / 2)^{+}} w_{1}(t) \\
&= \int_{0}^{\eta} \int_{\tau}^{(1+\eta) / 2} \phi_{p}^{-1} \\
& \times\left(\int_{s}^{(1+\eta) / 2} q(r) f\left(r, \frac{\sqrt{2}}{2} a r, \frac{\sqrt{2}}{2} a\right) \mathrm{d} r\right) \mathrm{d} s \mathrm{~d} \tau \\
&- \int_{(1+\eta) / 2}^{1} \int_{(1+\eta) / 2}^{\tau} \phi_{p}^{-1} \\
& \times\left(\int_{(1+\eta) / 2}^{s} q(r) f\left(r, \frac{\sqrt{2}}{2} a(1+\eta-r),-\frac{\sqrt{2}}{2} a\right) \mathrm{d} r\right) \mathrm{d} s \mathrm{~d} \tau \\
&=\left(\int_{0}^{(1+\eta) / 2}+\int_{(1+\eta) / 2}^{\eta}\right) \\
& \times \int_{\tau}^{(1+\eta) / 2} \phi_{p}^{-1}\left(\int_{s}^{(1+\eta) / 2} q(r) f\left(r, \frac{\sqrt{2}}{2} a r, \frac{\sqrt{2}}{2} a\right) \mathrm{d} r\right) \mathrm{d} s \mathrm{~d} \tau \\
&- \int_{(1+\eta) / 2}^{1} \int_{(1+\eta) / 2}^{\tau} \phi_{p}^{-1} \\
& \times\left(\int_{(1+\eta) / 2}^{s} q(r)\right.
\end{aligned}
$$$$
\begin{array}{r}
\left.\times f\left(r, \frac{\sqrt{2}}{2} a(1+\eta-r),-\frac{\sqrt{2}}{2} a\right) \mathrm{d} r\right) \mathrm{d} s \mathrm{~d} \tau \\
=\int_{0}^{(1+\eta) / 2} \int_{\tau}^{(1+\eta) / 2} \phi_{p}^{-1}
\end{array}
$$$$
\times\left(\int_{s}^{(1+\eta) / 2} q(r) f\left(r, \frac{\sqrt{2}}{2} a r, \frac{\sqrt{2}}{2} a\right) \mathrm{d} r\right) \mathrm{d} s \mathrm{~d} \tau
$$$$
+\int_{(1+\eta) / 2}^{\eta} \int_{\tau}^{(1+\eta) / 2} \phi_{p}^{-1}
$$$$
\times\left(\int_{s}^{(1+\eta) / 2} q(r) f\left(r, \frac{\sqrt{2}}{2} a r, \frac{\sqrt{2}}{2} a\right) \mathrm{d} r\right) \mathrm{d} s \mathrm{~d} \tau
$$$$
-\int_{(1+\eta) / 2}^{1} \int_{(1+\eta) / 2}^{\tau} \phi_{p}^{-1}
$$$$
\times\left(\int_{(1+\eta) / 2}^{s} q(r)\right.
$$$$
\left.\times f\left(r, \frac{\sqrt{2}}{2} a(1+\eta-r),-\frac{\sqrt{2}}{2} a\right) \mathrm{d} r\right) \mathrm{d} s \mathrm{~d} \tau
$$ 
Abstract and Applied Analysis

7

$$
\begin{aligned}
& =\int_{0}^{(1+\eta) / 2} \int_{\tau}^{(1+\eta) / 2} \phi_{p}^{-1} \\
& \quad \times\left(\int_{s}^{(1+\eta) / 2} q(r) f\left(r, \frac{\sqrt{2}}{2} a r, \frac{\sqrt{2}}{2} a\right) \mathrm{d} r\right) \mathrm{d} s \mathrm{~d} \tau \\
& =\lim _{t \rightarrow((1+\eta) / 2)^{-}} w_{1}(t) .
\end{aligned}
$$

Then (49) holds. Equation (50) can be obtained in a similar way. Thus from (49) and (50), it follows that

$$
w_{1}(t) \in C^{1}[0,1]
$$

We note that for $t \in[0,(1+\eta) / 2]$,

$$
\begin{aligned}
0 & \leq w_{1}(t) \\
& =\int_{0}^{t} \int_{\tau}^{(1+\eta) / 2} \phi_{p}^{-1} \\
& \times\left(\int_{s}^{(1+\eta) / 2} q(r) f\left(r, \frac{\sqrt{2}}{2} a r, \frac{\sqrt{2}}{2} a\right) \mathrm{d} r\right) \mathrm{d} s \mathrm{~d} \tau \\
& \leq \int_{0}^{t} \int_{\tau}^{(1+\eta) / 2} \phi_{p}^{-1}\left(\int_{s}^{(1+\eta) / 2} q(r) \phi_{p}\left(\frac{a}{A}\right) \mathrm{d} r\right) \mathrm{d} s \mathrm{~d} \tau \\
& \leq \frac{a}{A} \int_{0}^{t} \int_{\tau}^{(1+\eta) / 2} \phi_{p}^{-1}\left(\int_{0}^{(1+\eta) / 2} q(r) \mathrm{d} r\right) \mathrm{d} s \mathrm{~d} \tau \\
& \leq \frac{a}{A} A_{2} t=w_{0}(t),
\end{aligned}
$$

and for $t \in[(1+\eta) / 2,1]$,

$$
\begin{aligned}
& w_{1}(t) \\
& =\int_{0}^{\eta} \int_{\tau}^{(1+\eta) / 2} \phi_{p}^{-1} \\
& \quad \times\left(\int_{s}^{(1+\eta) / 2} q(r) f\left(r, \frac{\sqrt{2}}{2} a r, \frac{\sqrt{2}}{2} a\right) \mathrm{d} r\right) \mathrm{d} s \mathrm{~d} \tau \\
& -\int_{t}^{1} \int_{(1+\eta) / 2}^{\tau} \phi_{p}^{-1} \\
& \quad \times\left(\int_{(1+\eta) / 2}^{s} q(r)\right. \\
& \left.\quad \times f\left(r, \frac{\sqrt{2}}{2} a(1+\eta-r),-\frac{\sqrt{2}}{2} a\right) \mathrm{d} r\right) \mathrm{d} s \mathrm{~d} \tau
\end{aligned}
$$

$$
\begin{aligned}
\leq & \int_{0}^{\eta} \int_{\tau}^{(1+\eta) / 2} \phi_{p}^{-1}\left(\int_{s}^{(1+\eta) / 2} q(r) \phi_{p}\left(\frac{a}{A}\right) \mathrm{d} r\right) \mathrm{d} s \mathrm{~d} \tau \\
& +\int_{t}^{1} \int_{(1+\eta) / 2}^{\tau} \phi_{p}^{-1}\left(\int_{(1+\eta) / 2}^{s} q(r) \phi_{p}\left(\frac{a}{A}\right) \mathrm{d} r\right) \mathrm{d} s \mathrm{~d} \tau \\
\leq & \frac{a}{A} \int_{0}^{\eta} \int_{\tau}^{(1+\eta) / 2} \phi_{p}^{-1}\left(\int_{0}^{(1+\eta) / 2} q(r) \mathrm{d} r\right) \mathrm{d} s \mathrm{~d} \tau \\
& +\frac{a}{A} \int_{t}^{1} \int_{(1+\eta) / 2}^{\tau} \phi_{p}^{-1}\left(\int_{(1+\eta) / 2}^{1} q(r) \mathrm{d} r\right) \mathrm{d} s \mathrm{~d} \tau \\
\leq & \frac{a}{A} \int_{0}^{\eta} \int_{\tau}^{(1+\eta) / 2} A_{2} \mathrm{~d} s \mathrm{~d} \tau \\
& +\frac{a}{A} \int_{t}^{1} \int_{(1+\eta) / 2}^{\tau} \phi_{p}^{-1}\left(\int_{0}^{(1+\eta) / 2} q(r) \mathrm{d} r\right) \mathrm{d} s \mathrm{~d} \tau \\
\leq & \frac{a}{A} A_{2} \eta+\frac{a}{A} A_{0}^{\eta} A_{2}(1-t)=w_{0}(t) . \\
\hline & \mathrm{d} \tau+\int_{t}^{1} \int_{(1+\eta) / 2}^{\tau} A_{2} \mathrm{~d} s \mathrm{~d} \tau
\end{aligned}
$$

So,

$$
w_{1}(t) \leq w_{0}(t) \leq \frac{\sqrt{2}}{2} a, \quad t \in[0,1]
$$

Thus,

$$
\alpha\left(w_{1}\right):=\max _{0 \leq t \leq 1}\left|w_{1}(t)\right| \leq \frac{\sqrt{2}}{2} a .
$$

From assumptions, for $t \in[0,(1+\eta) / 2]$,

$$
\begin{aligned}
\left|w_{1}^{\prime}(t)\right| & =\int_{t}^{(1+\eta) / 2} \phi_{p}^{-1} \\
& \times\left(\int_{s}^{(1+\eta) / 2} q(r) f\left(r, \frac{\sqrt{2}}{2} a r, \frac{\sqrt{2}}{2} a\right) \mathrm{d} r\right) \mathrm{d} s \\
& \leq \int_{t}^{(1+\eta) / 2} \phi_{p}^{-1}\left(\int_{s}^{(1+\eta) / 2} q(r) \phi_{p}\left(\frac{a}{A}\right) \mathrm{d} r\right) \mathrm{d} s \\
& \leq \frac{a}{A} \int_{t}^{(1+\eta) / 2} \phi_{p}^{-1}\left(\int_{0}^{(1+\eta) / 2} q(r) \mathrm{d} r\right) \mathrm{d} s \\
& \leq \frac{a}{A} A_{2} \leq \frac{\sqrt{2}}{2} a=\left|w_{0}^{\prime}(t)\right|,
\end{aligned}
$$


and for $t \in[(1+\eta) / 2,1]$,

$$
\begin{aligned}
& \left|w_{1}^{\prime}(t)\right| \\
& =\mid \int_{(1+\eta) / 2}^{t} \phi_{p}^{-1}\left(\int_{(1+\eta) / 2}^{s} q(r)\right. \\
& \left.\times f\left(r, \frac{\sqrt{2}}{2} a(1+\eta-r),-\frac{\sqrt{2}}{2} a\right) \mathrm{d} r\right) \mathrm{d} s \mid \\
& \leq \int_{(1+\eta) / 2}^{t} \phi_{p}^{-1}\left(\int_{(1+\eta) / 2}^{1} q(r) \phi_{p}\left(\frac{a}{A}\right) \mathrm{d} r\right) \mathrm{d} s \\
& \leq \frac{a}{A} \int_{(1+\eta) / 2}^{t} \phi_{p}^{-1}\left(\int_{0}^{(1+\eta) / 2} q(r) \mathrm{d} r\right) \mathrm{d} s \\
& \leq \frac{a}{A} A_{2} \leq \frac{\sqrt{2}}{2} a=\left|w_{0}^{\prime}(t)\right| .
\end{aligned}
$$

Hence from (57) and (58), we have

$$
\beta\left(w_{1}\right):=\max _{0 \leq t \leq 1}\left|w_{1}^{\prime}(t)\right| \leq \frac{\sqrt{2}}{2} a .
$$

Consequently, from (56) and (59), it follows that

$$
\left\|w_{1}\right\| \leq \sqrt{2} \max \left\{\alpha\left(w_{1}\right), \beta\left(w_{1}\right)\right\} \leq a .
$$

From the proof of Lemma 3, we see that $w_{1}$ is nonnegative, concave, and pseudosymmetric about $\eta$ on $[0,1]$, and hence

$$
w_{1} \in \bar{P}_{a}
$$

Define $\left\{w_{n}\right\}$ as follows:

$$
w_{n+1}=T w_{n}=T^{n} w_{1}=T^{n+1} w_{0}, \quad n=0,1, \ldots
$$

Then $\left\{w_{n}\right\}$ is well defined and for $n=1,2, \ldots$,

$$
w_{n+1}(t) \leq w_{n}(t), \quad\left|w_{n+1}^{\prime}(t)\right| \leq\left|w_{n}^{\prime}(t)\right|, \quad t \in[0,1] . \quad(63)
$$

In fact, for $t \in[0,(1+\eta) / 2]$,

$$
\begin{aligned}
& w_{2}(t)=T w_{1}(t) \\
& =\int_{0}^{t} \int_{\tau}^{(1+\eta) / 2} \phi_{p}^{-1} \\
& \times\left(\int_{s}^{(1+\eta) / 2} q(r)\right. \\
& \left.\times f\left(r, w_{1}(r), w_{1}^{\prime}(r)\right) \mathrm{d} r\right) \mathrm{d} s \mathrm{~d} \tau \\
& \leq \int_{0}^{t} \int_{\tau}^{(1+\eta) / 2} \phi_{p}^{-1} \\
& \times\left(\int_{s}^{(1+\eta) / 2} q(r)\right. \\
& \left.\times f\left(r, \frac{\sqrt{2}}{2} a r, \frac{\sqrt{2}}{2} a\right) \mathrm{d} r\right) \mathrm{d} s \mathrm{~d} \tau \\
& =w_{1}(t), \\
& \left|w_{2}^{\prime}(t)\right|=\left|T w_{1}^{\prime}(t)\right| \\
& =\int_{t}^{(1+\eta) / 2} \phi_{p}^{-1} \\
& \times\left(\int_{s}^{(1+\eta) / 2} q(r)\right. \\
& \left.\times f\left(r, w_{1}(r), w_{1}^{\prime}(r)\right) \mathrm{d} r\right) \mathrm{d} s \\
& \leq \int_{t}^{(1+\eta) / 2} \phi_{p}^{-1} \\
& \times\left(\int_{s}^{(1+\eta) / 2} q(r)\right. \\
& \left.\times f\left(r, \frac{\sqrt{2}}{2} a r, \frac{\sqrt{2}}{2} a\right) \mathrm{d} r\right) \mathrm{d} s \\
& =w_{1}^{\prime}(t) .
\end{aligned}
$$

For $t \in[(1+\eta) / 2,1]$, since $w_{1}, w_{2} \in \bar{P}_{a}$, it follows from (64) and (65) that

$$
\begin{gathered}
w_{2}(t)=w_{2}(1+\eta-t) \leq w_{1}(1+\eta-t)=w_{1}(t), \\
\left|w_{2}^{\prime}(t)\right|=\left|w_{2}^{\prime}(1+\eta-t)\right| \leq\left|w_{1}^{\prime}(1+\eta-t)\right|=\left|w_{1}^{\prime}(t)\right| .
\end{gathered}
$$

So from (64)-(67), we have

$$
w_{2}(t) \leq w_{1}(t), \quad\left|w_{2}^{\prime}(t)\right| \leq\left|w_{1}^{\prime}(t)\right|, \quad t \in[0,1],
$$

that is, (63) holds when $n=1$. Assume that (63) holds when $n=k$, that is,

$$
w_{k+1}(t) \leq w_{k}(t), \quad\left|w_{k+1}^{\prime}(t)\right| \leq\left|w_{k}^{\prime}(t)\right|, \quad t \in[0,1] .
$$


Then from Lemma 5, we obtain

$$
\begin{aligned}
w_{k+2}(t) & =\left(T w_{k+1}\right)(t) \leq\left(T w_{k}\right)(t) \\
& =w_{k+1}(t), \quad t \in[0,1], \\
\left|w_{k+2}^{\prime}(t)\right| & =\left|\left(T w_{k+1}\right)^{\prime}(t)\right| \leq\left|\left(T w_{k}\right)^{\prime}(t)\right| \\
& =\left|w_{k+1}^{\prime}(t)\right|, \quad t \in[0,1] .
\end{aligned}
$$

So by induction (63) holds.

Since $T: \bar{P}_{a} \rightarrow \bar{P}_{a}$ is completely continuous, it follows that $\left\{w_{n}\right\}_{n=1}^{\infty}$ is relative compact. Then $\left\{w_{n}\right\}$ has a convergent subsequence $\left\{w_{n_{k}}\right\}$ and $w^{*} \in \bar{P}_{a}$ such that

$$
w_{n_{k}} \longrightarrow w^{*} \quad(k \longrightarrow \infty)
$$

that is,

$$
\begin{gathered}
w_{n_{k}}(t) \rightrightarrows w^{*}(t) \quad(k \longrightarrow \infty) \\
w_{n_{k}}^{\prime}(t) \rightrightarrows w^{* \prime}(t) \quad(k \longrightarrow \infty) \text { on }[0,1] .
\end{gathered}
$$

While from (63) and the fact for each $n=1,2, \ldots, w_{n}^{\prime}((1+$ $\eta) / 2)=0$ and $w_{n}^{\prime \prime}(t) \leq 0$ on $[0,1]$, it follows that

$$
\begin{array}{r}
\begin{array}{r}
w_{1}(t) \geq w_{2}(t) \geq \cdots \geq w_{n}(t) \geq w_{n+1}(t) \geq \cdots, \\
n=1,2, \ldots, \text { on }[0,1], \\
w_{1}^{\prime}(t) \geq w_{2}^{\prime}(t) \geq \cdots \geq w_{n}^{\prime}(t) \geq w_{n+1}^{\prime}(t) \geq \cdots, \\
n=1,2, \ldots, \text { on }\left[0, \frac{1+\eta}{2}\right], \\
w_{1}^{\prime}(t) \leq w_{2}^{\prime}(t) \leq \cdots \leq w_{n}^{\prime}(t) \leq w_{n+1}^{\prime}(t) \leq \cdots, \\
n=1,2, \ldots, \text { on }\left[\frac{1+\eta}{2}, 1\right] .
\end{array}
\end{array}
$$

Hence,

$$
\begin{gathered}
w_{n}(t) \rightrightarrows w^{*}(t) \quad(n \longrightarrow \infty), \\
w_{n}^{\prime}(t) \rightrightarrows w^{* \prime}(t) \quad(n \longrightarrow \infty) \text { on }[0,1]
\end{gathered}
$$

that is,

$$
w_{n} \longrightarrow w^{*}(n \longrightarrow \infty) \text {. }
$$

This together with the continuity of $T$ and $w_{n+1}=T w_{n}$, implies that

$$
T w^{*}=w^{*} .
$$

Also, since

$$
\begin{aligned}
& \begin{array}{l}
0 \leq w_{n}(t) \leq w_{0}(t) \\
= \begin{cases}\frac{\sqrt{2}}{2} a t, & 0 \leq t \leq \frac{1+\eta}{2}, \\
\frac{\sqrt{2}}{2} a(1+\eta-t), & \frac{1+\eta}{2} \leq t \leq 1,\end{cases} \\
0 \leq\left|w_{n}^{\prime}(t)\right| \leq\left|w_{1}^{\prime}(t)\right| \leq \frac{\sqrt{2}}{2} a, \quad t \in[0,1],
\end{array}
\end{aligned}
$$

we have

$$
0 \leq w^{*}(t) \leq \frac{\sqrt{2}}{2} a, \quad 0 \leq\left|w^{* \prime}(t)\right| \leq \frac{\sqrt{2}}{2} a, \quad t \in[0,1] .
$$

Furthermore, we have

$$
w^{*}(t)>0, \quad t \in(0,1] .
$$

In fact, from $\left(\mathrm{H}_{3}\right)$ and $w^{*}(t) \not \equiv 0$ on $[0,1]$, we have $w^{*}((1+$ $\eta) / 2)>0$. Since $w^{*}(t)$ is concave on $[0,1]$, then

$$
\begin{aligned}
w^{*}(t) & \geq \frac{w^{*}((1+\eta) / 2)-0}{((1+\eta) / 2)-0} t \\
& =\frac{2}{1+\eta} w^{*}\left(\frac{1+\eta}{2}\right) t>0, \quad t \in\left(0, \frac{1+\eta}{2}\right] .
\end{aligned}
$$

Consequently from the fact $w^{*}$ is pseudosymmetric on $[0,1]$, we have

$$
w^{*}(t)>0, \quad t \in(0,1]
$$

Let $v_{0}(t) \equiv 0$ on $[0,1]$, then $v_{0} \in \bar{P}_{a}$. Set $v_{n+1}=T v_{n}, n=0,1$, $2, \ldots$. Then from Lemma 6 , it follows that

$$
v_{n} \in \bar{P}_{a}, \quad n=1,2, \ldots
$$

From Lemma 4 , we see that $\left\{v_{n}\right\}_{n=1}^{\infty}$ is relative compact, and hence there exists a convergent subsequence $\left\{v_{n_{k}}\right\} \subset\left\{v_{n}\right\}$ and $v^{*} \in \bar{P}_{a}$ such that

$$
v_{n_{k}} \longrightarrow v^{*} \quad(k \longrightarrow \infty)
$$

that is,

$$
\begin{aligned}
& v_{n_{k}}(t) \rightrightarrows v^{*}(t) \quad(k \longrightarrow \infty) \text { on }[0,1] \\
& v_{n_{k}}^{\prime}(t) \rightrightarrows v^{* \prime}(t) \quad(k \longrightarrow \infty) \text { on }[0,1]
\end{aligned}
$$

Since $v_{1}=T v_{0}=T 0 \in \bar{P}_{a}$, then

$$
\begin{gathered}
v_{1}(t)=T v_{0}(t)=(T 0)(t) \geq 0, \quad t \in[0,1], \\
\left|v_{1}^{\prime}(t)\right|=\left|\left(T v_{0}\right)^{\prime}(t)\right|=\left|(T 0)^{\prime}(t)\right| \geq 0, \quad t \in[0,1] .
\end{gathered}
$$

Thus from Lemma 5,

$$
\begin{gathered}
v_{2}(t)=T v_{1}(t) \geq T v_{0}(t)=v_{1}(t), \quad t \in[0,1] \\
\left|v_{2}^{\prime}(t)\right|=\left|\left(T v_{1}\right)^{\prime}(t)\right| \geq\left|\left(T v_{0}\right)^{\prime}(t)\right|=\left|v_{1}^{\prime}(t)\right|, \quad t \in[0,1] .
\end{gathered}
$$

By induction, it is easy to see that for $n=1,2, \ldots$,

$$
\begin{gathered}
v_{n+1}(t) \geq v_{n}(t), \quad t \in[0,1], \\
\left|v_{n+1}^{\prime}(t)\right| \geq\left|v_{n}^{\prime}(t)\right|, \quad t \in[0,1] .
\end{gathered}
$$


From (84)-(89), we see that

$$
\begin{gathered}
v_{n}(t) \rightrightarrows v^{*}(t) \quad(n \longrightarrow \infty), \\
v_{n}^{\prime}(t) \rightrightarrows v^{* \prime}(t) \quad(n \longrightarrow \infty) \text { on }[0,1] .
\end{gathered}
$$

Therefore, $v_{n} \rightarrow v^{*}(n \rightarrow \infty), v^{*} \in \bar{P}_{a}$. By the continuity of $T$ and $v_{n+1}=T v_{n}$, we have

$$
T v^{*}=v^{*}
$$

Again from $\left(\mathrm{H}_{3}\right)$, we have $v^{*}(t)>0$ on $(0,1]$.

Since every fixed point of $T$ in $P$ is the solution of BVP (9), (10), then $w^{*}$ and $v^{*}$ are two positive, concave and pseudosymmetric solutions of BVP (9), (10). This completes the proof of the theorem.

\section{An Example}

Consider the following third-order four-point boundary value problem:

$$
\begin{gathered}
u^{\prime \prime \prime}(t)+f\left(t, u(t), u^{\prime}(t)\right)=0, \quad t \in(0,1), \\
u(0)=0, \quad u(1)=u\left(\frac{1}{2}\right), \quad u^{\prime \prime}\left(\frac{3}{4}\right)=0,
\end{gathered}
$$

where

$$
\begin{gathered}
f(t, u, v)=\frac{\sqrt{2}}{84}\left(\frac{3}{4}-t\right)\left(u v^{2}+16 \sqrt{2}\right), \\
(t, u, v) \in[0,1] \times[0,+\infty) \times \mathbb{R} .
\end{gathered}
$$

It is easy to see that BVP (92) corresponds to BVP (9), (10) when $p=2, q(t) \equiv 1$, and $\eta=1 / 2$. Take $a=6 \sqrt{2}$, and then $A=3 \sqrt{2} / 4$.

Next we verify that all conditions of Theorem 7 are satisfied. In fact, obviously the conditions $\left(\mathrm{H}_{0}\right),\left(\mathrm{H}_{1}\right),\left(\mathrm{H}_{2}\right)$, and $\left(\mathrm{H}_{3}\right)$ hold. In addition, for $0 \leq t \leq 3 / 4,0 \leq u_{1} \leq u_{2} \leq$ $6 \sqrt{2}, 0 \leq\left|v_{1}\right| \leq\left|v_{2}\right| \leq 6 \sqrt{2}$,

$$
\begin{gathered}
f\left(t, u_{1}, v_{1}\right) \leq f\left(t, u_{2}, v_{2}\right), \\
\max _{0 \leq t \leq 3 / 4} f(t, a, a)=f(0,6 \sqrt{2}, 6 \sqrt{2})=8=\phi_{2}\left(\frac{a}{A}\right) .
\end{gathered}
$$

Hence, from Theorem 7, BVP (92) has two positive, concave, and pseudosymmetric solutions $w^{*}$ and $v^{*}$ such that

$$
\begin{gathered}
0<w^{*}(t) \leq 6, \quad 0<\left|w^{* \prime}(t)\right| \leq 6, \quad t \in[0,1], \\
\lim _{n \rightarrow \infty} w_{n}=\lim _{n \rightarrow \infty} T^{n} w_{0}=w^{*}, \\
\lim _{n \rightarrow \infty} w_{n}^{\prime}=\lim _{n \rightarrow \infty}\left(T^{n} w_{0}\right)^{\prime}=w^{* \prime},
\end{gathered}
$$

where

$$
w_{0}(t)= \begin{cases}6 t, & 0 \leq t \leq \frac{3}{4} \\ 9-6 t, & \frac{3}{4} \leq t \leq 1\end{cases}
$$

for $n=0,1,2, \ldots$,

$$
\begin{aligned}
& w_{n+1}(t)=\left\{\begin{array}{c}
\int_{0}^{t} \int_{\tau}^{3 / 4} \int_{s}^{3 / 4} \frac{\sqrt{2}}{84}\left(\frac{3}{4}-r\right) \\
\quad \times\left(w_{n}(r) w_{n}^{\prime 2}(r)+16 \sqrt{2}\right) \mathrm{d} r \mathrm{~d} s \mathrm{~d} \tau, \\
\int_{0}^{1 / 2} \int_{\tau}^{3 / 4} \int_{s}^{3 / 4} \frac{\sqrt{2}}{84}\left(\frac{3}{4}-r\right) \\
\times\left(w_{n}(r) w_{n}^{\prime 2}(r)+16 \sqrt{2}\right) \mathrm{d} r \mathrm{~d} s \mathrm{~d} \tau \\
-\int_{t}^{1} \int_{3 / 4}^{\tau} \int_{3 / 4}^{s} \frac{\sqrt{2}}{84}\left(\frac{3}{4}-r\right) \\
\end{array}\right. \\
& 0<v^{*}(t) \leq 6 \sqrt{2}, \quad 0<\left|v^{* \prime}(t)\right| \leq 6 \sqrt{2}, \quad t \in[0,1], \\
& \lim _{n \rightarrow \infty} v_{n}=\lim _{n \rightarrow \infty} T^{n} v_{0}=v^{*}, \quad \lim _{n \rightarrow \infty} v_{n}^{\prime}=\lim _{n \rightarrow \infty}\left(T^{n} v_{0}\right)^{\prime}=v^{* \prime},
\end{aligned}
$$

where $v_{0}(t) \equiv 0$ on $[0,1]$ and for $n=0,1,2, \ldots$,

$$
v_{n+1}(t)=\left\{\begin{array}{cc}
\int_{0}^{t} \int_{\tau}^{3 / 4} \int_{s}^{3 / 4} \frac{\sqrt{2}}{84}\left(\frac{3}{4}-r\right) \\
\quad \times\left(v_{n}(r) v_{n}^{\prime 2}(r)+16 \sqrt{2}\right) \mathrm{d} r \mathrm{~d} s \mathrm{~d} \tau \\
\int_{0}^{1 / 2} \int_{\tau}^{3 / 4} \int_{s}^{3 / 4} \frac{\sqrt{2}}{84}\left(\frac{3}{4}-r\right) \\
\quad \times\left(v_{n}(r) v_{n}^{\prime 2}(r)+16 \sqrt{2}\right) \mathrm{d} r \mathrm{~d} s \mathrm{~d} \tau \\
-\int_{t}^{1} \int_{3 / 4}^{\tau} \int_{3 / 4}^{s} \frac{\sqrt{2}}{84}\left(\frac{3}{4}-r\right) \\
\times\left(v_{n}(r) v_{n}^{\prime 2}(r)+16 \sqrt{2}\right) \mathrm{d} r \mathrm{~d} s \mathrm{~d} \tau
\end{array}\right.
$$

The first two terms of $\left\{w_{n}(t)\right\}$ and three terms of $\left\{v_{n}(t)\right\}$, respectively, are as follows:

$$
w_{0}(t)= \begin{cases}6 t, & 0 \leq t \leq \frac{3}{4} \\ 9-6 t, & \frac{3}{4} \leq t \leq 1\end{cases}
$$




$$
w_{1}(t)=\left\{\begin{aligned}
- & \frac{3 \sqrt{2}}{70} t^{5}+\left(\frac{9 \sqrt{2}}{112}-\frac{1}{63}\right) t^{4}+\frac{1}{21} t^{3} \\
- & \left(\frac{81 \sqrt{2}}{896}+\frac{3}{56}\right) t^{2} \\
& +\left(\frac{243 \sqrt{2}}{3584}+\frac{3}{112}\right) t, \quad 0 \leq t \leq \frac{3}{4}, \\
\frac{3 \sqrt{2}}{70} t^{5}-\left(\frac{27 \sqrt{2}}{112}+\frac{1}{63}\right) t^{4} & +\left(\frac{27 \sqrt{2}}{56}+\frac{1}{21}\right) t^{3} \\
v_{1}(t)= & \left.-\frac{405 \sqrt{2}}{63}+\frac{3}{56}\right) t^{4}+\frac{1}{21} t^{3}-\frac{3}{56} t^{2}+\frac{3}{112} t, \quad t \in[0,1], \\
& -\frac{729 \sqrt{2}}{35840}, \quad \frac{3}{4} \leq t \leq 1 ; \\
v_{2}(t)= & \frac{\sqrt{2}}{2867038902} t^{14}-\frac{3}{273051324} t^{13} \\
& +\frac{\sqrt{2}}{56010528} t^{12}-\frac{\sqrt{2}}{18670176} t^{11} \\
& +\frac{47 \sqrt{2}}{426746880} t^{10}-\frac{65 \sqrt{2}}{398297088} t^{9} \\
& +\frac{\sqrt{2}}{5619712} t^{8}-\frac{59 \sqrt{2}}{413048832} t^{7} \\
& +\frac{13 \sqrt{2}}{157351936} t^{6}-\frac{51 \sqrt{2}}{1573519360} t^{5} \\
& +\left(\frac{9 \sqrt{2}}{1258815488}-\frac{1}{63}\right) t^{4}+\frac{1}{21} t^{3} \\
& \left(\frac{81 \sqrt{2}}{598476849152}+\frac{3}{112}\right) t, \quad t \in[0,1] .
\end{aligned}\right.
$$

\section{Acknowledgment}

This work was supported by the NSFC (11126339 and 11201008).

\section{References}

[1] M. Greguš, Third Order Linear Differential Equations, D. Reidel, Dordrecht, The Netherlands, 1987.

[2] A. R. Aftabizadeh, C. P. Gupta, and J.-M. Xu, "Existence and uniqueness theorems for three-point boundary value problems," SIAM Journal on Mathematical Analysis, vol. 20, no. 3, pp. 716-726, 1989.
[3] R. P. Agarwal, "Existence-uniqueness and iterative methods for third-order boundary value problems," Journal of Computational and Applied Mathematics, vol. 17, no. 3, pp. 271-289, 1987.

[4] D. R. Anderson and J. M. Davis, "Multiple solutions and eigenvalues for third-order right focal boundary value problems," Journal of Mathematical Analysis and Applications, vol. 267, no. 1, pp. 135-157, 2002.

[5] Z. Bai and X. Fei, "Existence of triple positive solutions for a third order generalized right focal problem," Mathematical Inequalities \& Applications, vol. 9, no. 3, pp. 437-444, 2006.

[6] A. Cabada and S. Lois, "Existence of solution for discontinuous third order boundary value problems," Journal of Computational and Applied Mathematics, vol. 110, no. 1, pp. 105-114, 1999.

[7] A. Cabada, M. R. Grossinho, and F. Minhós, "Extremal solutions for third-order nonlinear problems with upper and lower solutions in reversed order," Nonlinear Analysis. Theory, Methods \& Applications, vol. 62, no. 6, pp. 1109-1121, 2005.

[8] J. Chu and Z. Zhou, "Positive solutions for singular nonlinear third-order periodic boundary value problems," Nonlinear Analysis. Theory, Methods \& Applications, vol. 64, no. 7, pp. 1528-1542, 2006.

[9] Z. Du, W. Ge, and X. Lin, "Existence of solutions for a class of third-order nonlinear boundary value problems," Journal of Mathematical Analysis and Applications, vol. 294, no. 1, pp. 104112,2004

[10] P. W. Eloe and J. Henderson, "Uniqueness implies existence and uniqueness conditions for nonlocal boundary value problems for $n$th order differential equations," Journal of Mathematical Analysis and Applications, vol. 331, no. 1, pp. 240-247, 2007.

[11] J. R. Graef and J. R. L. Webb, "Third order boundary value problems with nonlocal boundary conditions," Nonlinear Analysis. Theory, Methods \& Applications, vol. 71, no. 5-6, pp. 1542-1551, 2009.

[12] M. D. R. Grossinho, F. M. Minhós, and A. I. Santos, "Existence result for a third-order ODE with nonlinear boundary conditions in presence of a sign-type Nagumo control," Journal of Mathematical Analysis and Applications, vol. 309, no. 1, pp. 271283, 2005.

[13] C. P. Gupta and V. Lakshmikantham, "Existence and uniqueness theorems for a third-order three-point boundary value problem," Nonlinear Analysis. Theory, Methods \& Applications, vol. 16, no. 11, pp. 949-957, 1991.

[14] J. Henderson, "Best interval lengths for boundary value problems for third order Lipschitz equations," SIAM Journal on Mathematical Analysis, vol. 18, no. 2, pp. 293-305, 1987.

[15] G. Infante and P. Pietramala, "A third order boundary value problem subject to nonlinear boundary conditions," Mathematica Bohemica, vol. 135, no. 2, pp. 113-121, 2010.

[16] D. Jiang and R. P. Agarwal, "A uniqueness and existence theorem for a singular third-order boundary value problem on $[0, \infty)$," Applied Mathematics Letters, vol. 15, no. 4, pp. 445-451, 2002.

[17] S. Jin and S. Lu, "Existence of solutions for a third-order multipoint boundary value problem with $p$-Laplacian," Journal of the Franklin Institute. Engineering and Applied Mathematics, vol. 347, no. 3, pp. 599-606, 2010.

[18] Y. Li, "Positive periodic solutions for fully third-order ordinary differential equations," Computers \& Mathematics with Applications, vol. 59, no. 11, pp. 3464-3471, 2010.

[19] R. Ma, "Multiplicity results for a third order boundary value problem at resonance," Nonlinear Analysis. Theory, Methods \& Applications, vol. 32, no. 4, pp. 493-499, 1998. 
[20] F. M. Minhós, "On some third order nonlinear boundary value problems: existence, location and multiplicity results," Journal of Mathematical Analysis and Applications, vol. 339, no. 2, pp. 1342-1353, 2008.

[21] D. J. O'Regan, “Topological transversality: applications to third order boundary value problems," SIAM Journal on Mathematical Analysis, vol. 18, no. 3, pp. 630-641, 1987.

[22] M. Pei and S. K. Chang, "Existence and uniqueness of solutions for third-order nonlinear boundary value problems," Journal of Mathematical Analysis and Applications, vol. 327, no. 1, pp. 2335, 2007.

[23] I. Rachůnková, "Periodic boundary value problems for thirdorder differential equations," Mathematica Slovaca, vol. 41, no. 3, pp. 241-248, 1991.

[24] B. Sun, J. Zhao, P. Yang, and W. Ge, "Successive iteration and positive solutions for a third-order multipoint generalized right-focal boundary value problem with $p$-Laplacian," Nonlinear Analysis. Theory, Methods \& Applications, vol. 70, no. 1, pp. 220-230, 2009.

[25] J. Sun and Y. Liu, "Multiple positive solutions of singular thirdorder periodic boundary value problem," Acta Mathematica Scientia B, vol. 25, no. 1, pp. 81-88, 2005.

[26] Y. Wang and W. Ge, "Existence of solutions for a third order differential equation with integral boundary conditions," Computers \& Mathematics with Applications, vol. 53, no. 1, pp. 144154, 2007.

[27] P. J. Y. Wong, "Constant-sign solutions for a system of thirdorder generalized right focal problems," Nonlinear Analysis. Theory, Methods \& Applications, vol. 63, pp. 2153-2163, 2005.

[28] Y. Wu and Z. Zhao, "Positive solutions for third-order boundary value problems with change of signs," Applied Mathematics and Computation, vol. 218, no. 6, pp. 2744-2749, 2011.

[29] Q. Yao, "Successive iteration of positive solution for a discontinuous third-order boundary value problem," Computers \& Mathematics with Applications, vol. 53, no. 5, pp. 741-749, 2007.

[30] C. Zhou and D. Ma, "Existence and iteration of positive solutions for a generalized right-focal boundary value problem with p-Laplacian operator," Journal of Mathematical Analysis and Applications, vol. 324, no. 1, pp. 409-424, 2006.

[31] C. Yang and J. Yan, "Positive solutions for third-order SturmLiouville boundary value problems with $p$-Laplacian," Computers \& Mathematics with Applications, vol. 59, no. 6, pp. 20592066, 2010.

[32] R. Avery and J. Henderson, "Existence of three positive pseudosymmetric solutions for a one-dimensional p-Laplacian," Journal of Mathematical Analysis and Applications, vol. 277, no. 2, pp. 395-404, 2003.

[33] B. Sun and W. Ge, "Successive iteration and positive pseudosymmetric solutions for a three-point second-order $p$ Laplacian boundary value problems," Applied Mathematics and Computation, vol. 188, no. 2, pp. 1772-1779, 2007. 


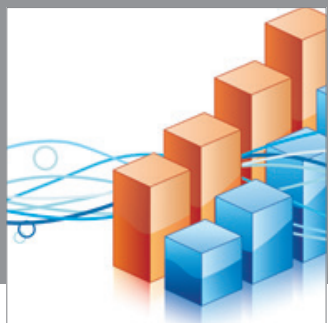

Advances in

Operations Research

mansans

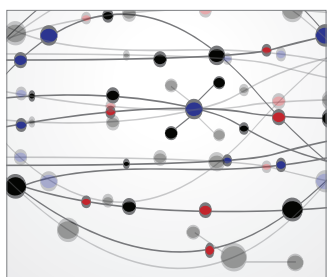

The Scientific World Journal
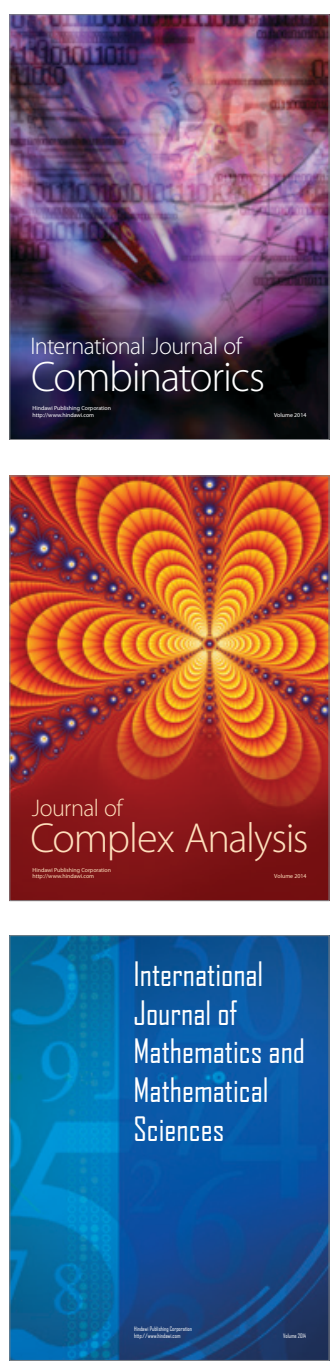
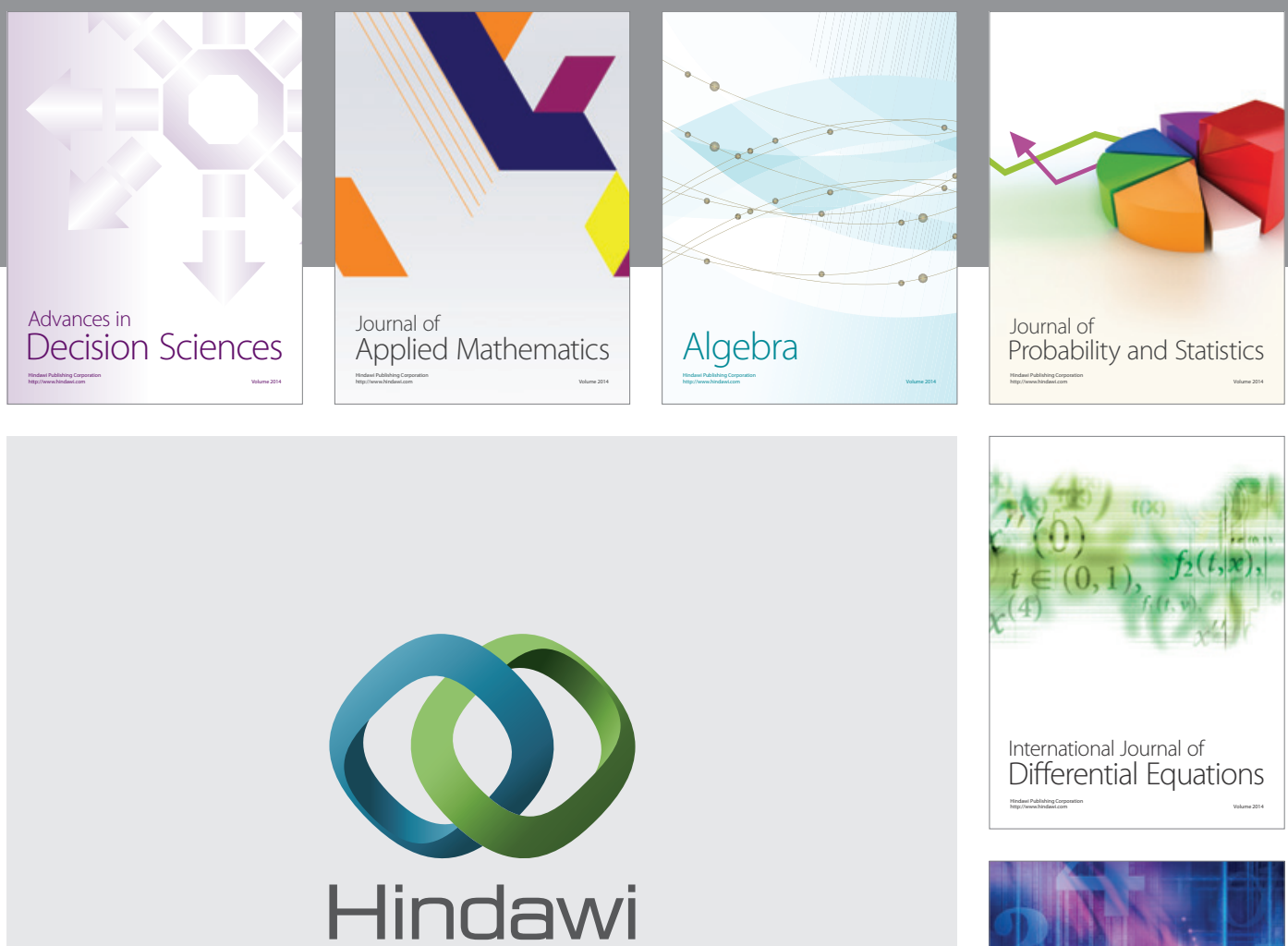

Submit your manuscripts at http://www.hindawi.com
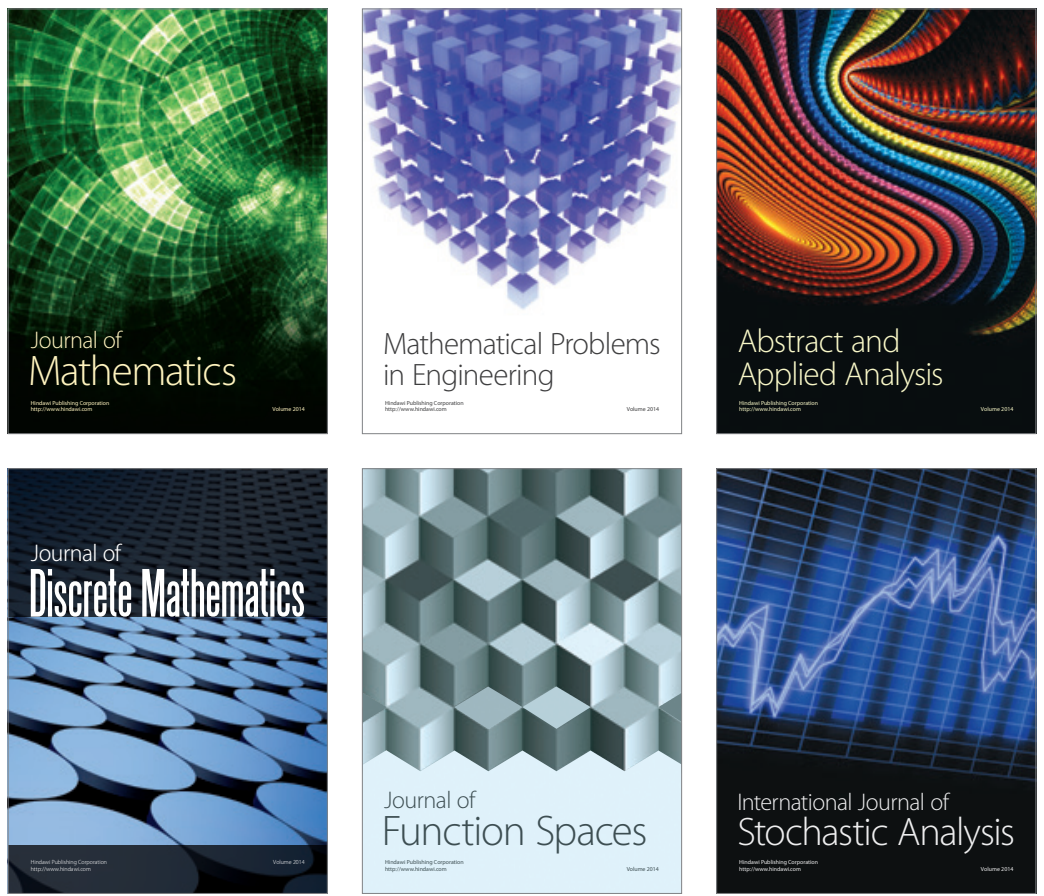

Journal of

Function Spaces

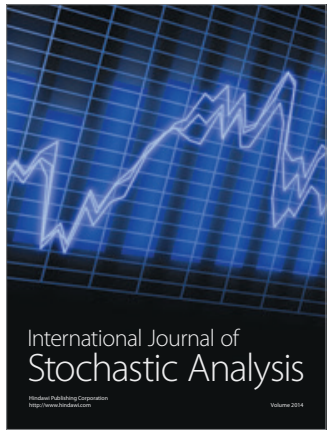

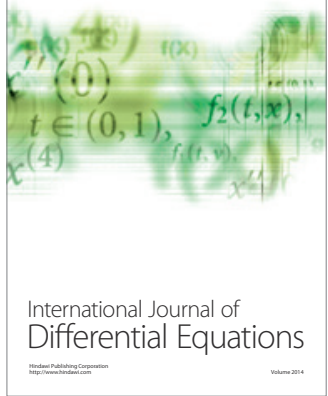
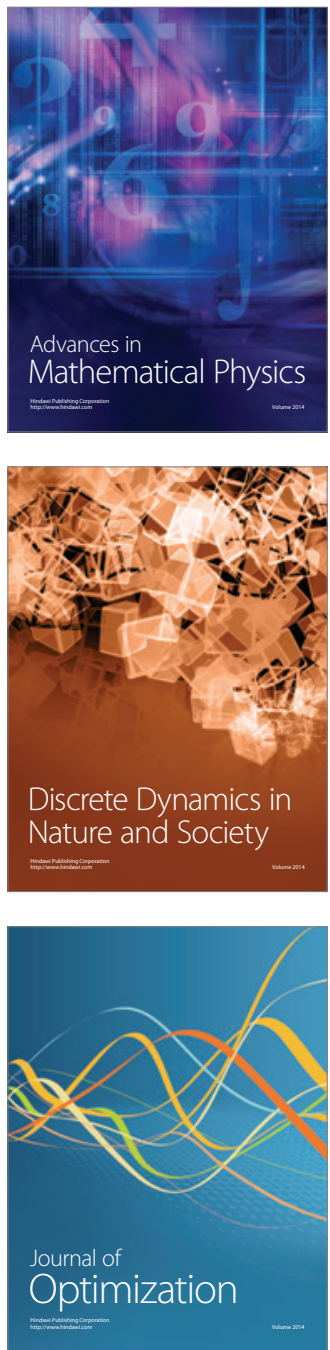